\title{
Tactile Ground Surface Indicator -Installation and challenges faced by visually impaired globally
}

\author{
J. Piriya Ram Prabhu, N. Lakshmi Thilagam
}

\begin{abstract}
Tactile Surfaces are used by visually impaired people globally as an assistive tool for tactile cues from the environment for their mobility. Tactile Ground Surface Indicators (TGSI) or Tactile Walking Surface Indicators (TWSI) are used in many countries for visually impaired as a standard tool for enabling a barrier-free environment. TGSI enable people with all types of visual impairment to wayfind, orient and detect hazard in the built environment. The purpose of this study is to find the challenges faced by the installation of tactile ground surface indicators and the challenges faced by visually impaired globally. This paper attempts to review the relevant literature based on both installations of tactile ground surface indicators and the issues faced by the visually impaired in many countries. The literature review shows that the visually impaired face challenges of wayfinding, orientation, and hazard warning in many countries because of the absence of standardized design of size, texture, color and installation protocol of TGSI. Maintenance and reinstallation of TGSI is an issue in many countries which causes confusion and discomfort to visually impaired.
\end{abstract}

Keywords-Orientation, Tactile Ground Surface Indicators, Tactile Walking Surface Indicators, Visually impaired, Wayfinding

\section{INTRODUCTION}

$\mathrm{W}$ orld Health Organisation (WHO) (2018) estimated that approximately 1.3 billion people all over the world are visually impaired [1]. People living with mild vision impairment are 188.5 million, people who have moderate to severe vision impairment are 217 million, and 36 million people are blind all around the world [2]. Every day visually impaired people have to access the environment around them. They have to wayfind and orient themselves in order to go by their daily routine in and out of the built environment. They have to detect and negotiate through places of risk. Tactile surfaces assist them to navigate and access their desired destination.

Tactile surfaces warn the visually impaired about the places of risk ahead. Visually impaired pedestrians use a long white cane to move around and find their way using tactile surfaces to negotiate through obstacles [3]. Visually impaired people also use the sole of their footwear to detect tactile surfaces. Tactile surfaces are used all over the world for the assistance of visually impaired to enable a barrierfree environment which has become a standard in many countries [3], [4], [10].

Revised Manuscript Received on December 09, 2019

* Correspondence Author

J. Piriya Ram Prabhu*, Research Scholar, Kalasalingam School of Architecture, Kalasalingam Academy for Research and Education, TamilNadu, India. Email: jprp10@gmail.com

Dr. (Prof) N.Lakshmi Thilagam, Director \& Professor, Kalasalingam School of Architecture, Kalasalingam Academy for Research and Education, TamilNadu, India. Email: hodbarch@klu.ac.in
The Tenji Blocks was invented by the Japanese inventor Seiichi Miyake. Tenji Blocks later became Tactile Ground Surface Indicators (TGSI)[4]. Tactile paving surface indicator, tactile paving, and tactile guide path are also the common names for TGSI [5]. The International Organization for Standardization (ISO) refers to these as Tactile Walking Surface Indicators [8], [15]. TGSI and TWSI are the same tactile blocks with the same purpose for the visually impaired people globally. TGSI is installed in many countries on sidewalks, to navigate and find intersections, crossings, and curbs. [3], [4], [5], [12], [13] TGSI is installed in streets, walk paths, malls, railway stations, public transport stations, and in many public places to navigate and detect entrances, stairs, ramps, and obstructions. The direction is guided using directional indicators which have parallel bars or stripes on the surface. Warnings are indicated by dots on the tactile surface of the warning indicators. [6], [7], [12], [13].

Spatial recognition is a key factor to find routes through known and unknown spaces. Visually impaired individuals need to have spatial judgment to move forward with comfort and confidence [16]. Visually impaired pedestrians in many countries face issues using TGSI or TWSI inside and outside the built environment due to the issues of installation. The challenges faced by the visually impaired are mostly due to the lack of standardization in the installation procedure and design of the tactile surfaces. The material, color, and sizes TGSI are different and this causes confusion and risk for visually impaired to recognize the space and move forward in the built environment [6], [7], [9], [10].

\section{TACTILE GROUND SURFACE INDICATORS}

TGSI is used in most of the countries to indicate direction and to alert the visually impaired in the built environment. The common types of TGSI blocks in many countries are direction blocks and warning blocks. The installation of these blocks is mandatory in most of the countries around the world for a barrier-free environment for visually impaired [5], [6], [7], [12], [13].

\section{A. Directional Blocks:}

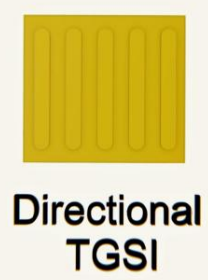

Fig.1. Directional TGSI Block 


\section{Source: Author}

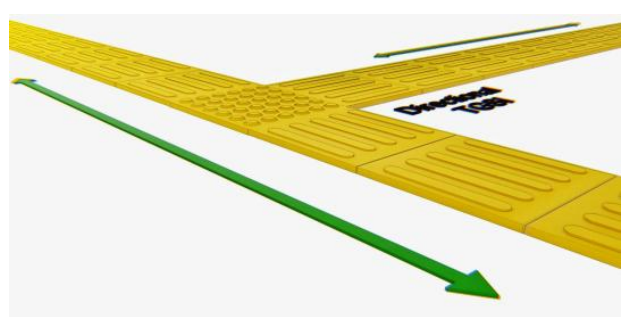

Fig.2. Indication of Directions Source: Author

In Fig.1, the directional Block surface has ribs which indicate the direction for visually impaired. In Fig.2 the blocks are placed in both indoor and outdoor for easy access and navigation. TGSI is placed at the appropriate locations in a street or inside a building to enable them to find direction towards their destination of travel. TGSI is used at cross junctions to orient the visually impaired [3], [5], [7], [12], [13].

\section{B. Warning TGSI Blocks:}

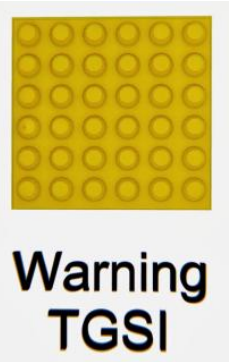

Fig.1. Warning TGSI Block

Source: Author

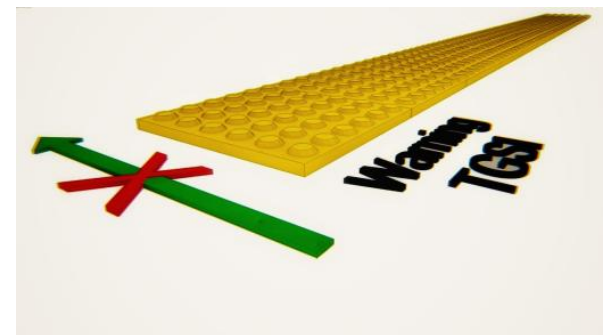

Fig.2. Indication of hazards

Source: Author

In Fig.3, the Warning Blocks surface has dots or truncated domes. In Fig.4, the blocks are placed to warn the visually impaired on the approaching hazards in their path. The use of warning blocks is important at pathway endings, platform edges, stairs, doorways and places of obstacles. These are mostly used in railway stations and places where streets cross over [3], [5], [7], [12], [13].

\section{GLOBAL INSTALLATIONS AND ISSUES}

The installation of TGSI has been an issue in many countries. The installation errors in 16 different countries were extensively surveyed by Mizuno et al [7]. The research was carried out in selected cities in America, Australia, Europe, and Aisa. All the countries have implemented a barrier-free environment for visually impaired and disabled. The findings of the survey clearly show that most of the countries follow an intermix rule on the procedure of installation methods of TGSI. The basic rule of installation was incorporated from the Japanese installation rules followed in Japan. Installation of TGSI all over the world is not the same but is as per the requirements of the country and culture. In the United Kingdom, directional blocks and warning blocks are both used as warning blocks. Warning Blocks are not used and directional blocks are mainly used in Germany and few countries.

The miss-match of TGSI installation is found in almost all countries globally. Visually impaired face challenges in these countries because of the inconsistency of installation standards. In [6] The installation of TGSI in Indonesia has issues of obstacles in the path of TGSI. Warning blocks and directional blocks are missing in the sidewalks to navigate them through the obstacles. Poles, parked vehicles, and vendors are on the path of TGSI. Visually impaired find it confusing and difficult to negotiate through the obstacles and reach their destination. Warning indicators are missing at the point of obstacles on the sidewalks. Reference [5] TGSI installation in China is based on the elements of Type, Pattern and visual contrast as per the standards from the department of transportation. TGSI used in China are both directional and hazard warning blocks. TGSI is not connected correctly and the maintenance is poor after installation. The installation guidelines are confused with guidelines from other countries [5].

\section{A. Issues in Colour of TGSI:}

TGSI block colours are important for people with low and moderate vision. Coloured TGSI is mostly used in railway stations and crosswalks in many countries. The colours enable them to additionally identify the blocks along with the tactile surface. Mizuno et al [7] study state that most of the countries use white, grey, yellow and black coloured TGSI blocks around the world. United Kingdom, America, Australia, Germany, and Korea predominantly use white yellow and black. Korea, Hong Kong, and China use brown along with other colours. Grey and silver coloured TGSI are also used in many countries.

Hong Kong and china uniquely use green coloured TGSI along with other colours [7]. TGSI colours and luminosity can be confusing for people with vision impairment because of the inconsistent colour standards followed globally. Standardization of Specific colours for specific TGSI is missing. Same colours are used for both warning and directional TGSI causes confusion [3], [7].

\section{B. Issues of Material and Size of TGSI:}

TGSI or TWSI blocks are made from natural and synthetic materials. The material used must be durable and slipresistant. Concrete and rubber are commonly used for manufacturing TGSI. Ceramic and metals are also used to make TGSI[3],[7].In Belgium, the installation methods used are unique where they use metal disks $(85 \mathrm{~mm}$ dia, $8 \mathrm{~mm}$ in height) that are comparatively larger than Japanese block protrusions (22mm dia, $5 \mathrm{~mm}$ height). The Japanese standard for the size of TGSI is followed in many countries. The Japanese TGSI size specifications do not hinder people in a wheelchair. The height of the Belgian Blocks made of metal causes issues for wheelchair users. Visually impaired people, Elderly 
pedestrians, and children find it to be slippery.

Similarly, a metal bar ( $3 \mathrm{~mm}$ height) has been embedded in some parts of Belgium city that is neither a hindrance/obstacle to wheelchair users nor helps the visually impaired people [7]. Australia and New Zealand follow the Japanese standard for TGSI size and material. In Germany, the blocks have thin linear protrusions and in the Netherlands, the blocks have a thin recessed line with very little surface irregularity, such blocks are extremely difficult to detect with the feet or white cane. The combination of TGSI made of different materials installed in transit stations, pedestrian walks paths creates a huge confusion for the people with impaired vision. The visually impaired are not able to detect and identify the path and face issues of faulty direction and orientation [3], [7], [12].

\section{Issues of Global standardization in Design and Installation:}

Designing of tactile surfaces has to consider the efficient medium in order to provide effective transfer of information. The integration of information to enhance the tactile factor is very prominent in order to ease the navigation part. Since the material chosen for tactility is considered as a medium, stronger the medium faster and easier information transfer is to the user [13], [14], [15]. The global usage of TGSI is predominantly at railway stations and at crossroads. Visually impaired people are not able to use TGSI efficiently at these places. The layout of the installation is not consistent. The color and texture are key factors for people with moderate visual impairment to identify TGSI. The contrast of luminance to the surrounding environment and surfaces can assist or impair the visibility of the tactile surfaces [9], [10], [11]. Japan railway stations are the places of maximum installation of tactile surfaces for passengers to orient and navigate inside the railway stations [9]. Errors in layout, texture, and color cause time delay and panic at peak hours when both people with vision and visually challenged have to negotiate. In Malaysia, the visually impaired had issues at Light Rail Transit (LRT) stations due to the placements of TGSI on the platforms mostly for hazard indications. The 90-degree corner issues were faced by the visually impaired which caused them issues of discomfort negotiating corners at LRT stations in Malaysia [11].

On evaluating the effectiveness of TGSI by $\emptyset_{\mathrm{v} s t e d a l}$ et al [14] identifies the tactile surface has to be integrated with information of the design using the surface material type, size, and color as a medium for carrying information for the user group. All navigation aspects depend upon the information provided by the surrounding context. In the case of a visually impaired person design of tactile material is very crucial for considering the safety aspects. The key considerations of TGSI by Mizuno et al [7] on the quality of material used for tactile, environmental factors have to be considered so that the durability and tactile factors are depended. So, consideration of design factors is not only the aspects but other elements too. With the consideration of indepth information regarding the material with responding to surrounding factors and visual information-based data, especially color system and tactile factors creating fewer disturbances from the outside environment. The general issues identified through these situations are the lack of incorporating texture of tactile material with its surrounding context which cannot be differentiated by the user group and the lack of universal regulation over the size of installation which is not addressed.

The errors in the installation of TGSI in many countries are based on identically following the installation rules followed in Japan. The Japanese rules of installation were copied without cross-checking the difference in situations prevailing in Japan and the other countries. The installation rules in Japan have errors and these errors reflected in the countries that followed the same rules of installation [7]. In China and Indonesia, Sidewalks are obstructed by many interventions such as bicycles, street vendors, etc which is a rare phenomenon in Japan [5], [6], [7]. In many cases, the warning blocks are misplaced instead of the required directional blocks and vice versa which further leads to the confusion of wayfinding for the visually impaired persons. Excessive installation of TGSI than specified standards leads to discomfort and difficulty in using the path [10], [13], [15].

\section{CONCLUSION}

Globally the standards and guidelines for a barrier-free environment for people with disabilities have been standardized and implemented. Visually impaired use various cues from the environment to orient and wayfind. Tactile cues from TGSI or TWSI enable the visually impaired to experience a barrier-free built environment. Japanese standards of TGSI design and installation are used in many countries. The countries following the Japanese installation rules face issues on the implementation of TGSI. Some countries have their own standards for the installation and design of TGSI. In a few countries, there is a difference in TGSI used in their cities. The lack of global installation standards, color, material and size cause confusion and discomfort for visually impaired.

TGSI design and installation have to take in consideration of persons on the wheelchair and elderly along with visually impaired users. Further study on the end-user experience and inputs are needed to upgrade TGSI. The countries using TGSI or TWSI together should formulate a Universal standard of design and installation. The awareness of TGSI should be imparted to the visually impaired in their basic skill development programs. Information on TGSI or TWSI used in a city or country can be introduced to visually impaired travelers on entering a new city or country. TGSI is yet to be installed in many countries around the world. The installation of TGSI or TWSI has to be made mandatory in all countries around the world to make the world a better place for people with visual impairment and disabilities.

\section{REFERENCES}

1. "Vision impairment and blindness'. Accessed 1 July 2019 https://www.who.int/news-room/fact-sheets/detail/blindness-and-visualimpairment

2. Bourne RRA, Flaxman SR, Braithwaite T, Cicinelli MV, Das A, Jonas JB, et al.; Vision Loss Expert Group, "Magnitude, temporal trends, and projections of the global prevalence of blindness and distance and near vision impairment: a systematic 
review and meta-analysis," Lancet Glob Health,5(9):e888-97, Sep 2017.

3. Antonio Lauria "Tactile pavings and urban places of cultural interest: A Study on detectability of contrasting walking surface materials," Journal of Urban Technology, 2017.

4. Josh Grisdale, and Accessible Japan. "How Japanese Inventor of Tenji Blocks Changed the Lives of Millions Around the World,". Japan Forward, 27 March 2019.

5. Kin Wai Michael Siu, "Design standard for inclusion: tactile ground surface indicators in China," Facilities, Vol. 31 Iss $7 / 8$ pp. 314 - 327, 2013.

6. Ardilson Pembuain, Sigit Priyanto, Latif Budi Suparma, "The evaluation of tactile ground surface indicator condition and effectiveness on the sidewalk in Yogyakarta City, Indonesia," IATSS Research, 2019 in press..

7. TomomiMIZUNO,ArisaNISHIDATE,KatsumiTOKUDA,KunijiroARAI , "Installation errors and corrections in tactile ground surface indicators in Europe, America, Oceania, and Asia," IATSS RESEARCH Vol.32 No.2, 2008.

8. ISO 23599:2019, "Assistive products for blind and vision impaired persons-Tactile walking surface indicators."

9. Hisato OHNO, Ayako SUZUKI, Naohiro AKIU, "Measuring methods of luminance contrast between tactile walking surface indicators and their surrounding or adjacent surfaces at railway stations," Quarterly Report of RTRI • May 2017

10. Vijaya Prakash R, Taduri S, "Safe navigation for elderly and visually impaired people using adhesive tactile walking surface indicators in home environment," Information and Communication Technology for Sustainable Development. Advances in Intelligent Systems and Computing, vol 933. Springer, June 2019.

11. Fairuzzana Ahamd Padzi, Fuziah Ibrahim, Norashikin Abdul Karim "Incongruent installation of tactile ground surface indicator toward visual impaired people's need: Masjid Jamek station," Procedia - Social and Behavioral Sciences 101 ( 2013 ) $130-139$.

12. Ståhl, Agneta, Emma Newman, Synneve Dahlin-Ivanoff, Mai Almén, and Susanne Iwarsson. "Detection of warning surfaces in pedestrian environments: The Importance for Blind People of Kerbs, Depth, and Structure of Tactile Surfaces," Disability and Rehabilitation 32, no. 6 (January 2010): 469-82.

13. Øvstedal, Liv Rakel, Terje Lindland, and Inger Marie Lid. "On our way establishing national guidelines on tactile surface indicators,". International Congress Series 1282 (September 2005): 1046-50.

14. Øvstedal, Liv Rakel, Inger Marie Lid, and Terje Lindland. "How to evaluate the effectiveness of a tactile surface indicator System? ," International Congress Series 1282 (September 2005): 1051-55.

15. Halime Demirkan, "Effectiveness of tactile surface indicators in 'design for all' context," Open House International. 38. 43-51., 2013.

16. C. Thinus-Blanc and F. Gaunet, "Representation of space in blind persons: Vision as a spatial sense?", Psychological Bulletin, vol. 121, no. 1, pp. 20-42, 1997.

\section{AUTHORS PROFILE}

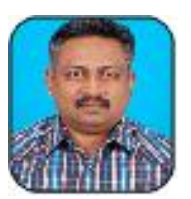

Ar. J. Piriya Ram Prabhu pursuing Ph.D. in Architecture from school of Architecture, Kalasalingam Academy for Research and Education, TamilNadu, India. He has acquired his B-arch. degree from Regional Engineering College Trichy, Tamilnadu and M-arch Degree from UNSW Sydney, Australia. He is a registered Architect with Council of Architecture, India and an associate member of Indian Institute of Architecture.

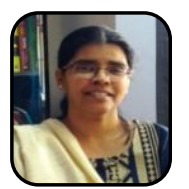

Dr. N. Lakshmi Thilagam is an Architect and Urban Designer and holds a PhD from the Indian Institute of Technology, Kharagpur, India. Her research interests are traditional urbanism, space syntax application for historic cities, heritage and urban conservation. She is currently conducting research to analyze the principles of sustainability exhibited in the traditional urban patterns of the historic cities of Tamil Nadu. As a passionate academician who has been teaching for the last 2 decades she also writes on the subject of Architectural education. 